\title{
Satisfação com a organização do cuidado em idosos usuários dos serviços avaliados pelo PMAQ
}

\author{
Satisfaction with the organization of care among elderly users of \\ the services assessed by the PMAQ
}

Juleimar Soares Coelho de Amorim (https://orcid.org/0000-0003-3218-1769) ${ }^{1}$

Luciana Barcellos Teixeira (https://orcid.org/0000-0003-1654-3723) ${ }^{2}$

Antônio Alcindo Ferla (https://orcid.org/0000-0002-9408-1504) ${ }^{2}$
${ }^{1}$ Instituto Federal de Educação, Ciência e Tecnologia do Rio de Janeiro. R. Prof. Carlos Wenceslau 343, Realengo. 21710-240 Rio de Janeiro RJ Brasil.

juleimar@yahoo.com.br ${ }^{2}$ Programa de PósGraduação em Saúde Coletiva, Escola de Enfermagem, Universidade Federal do Rio Grande do Sul. Porto Alegre RS Brasil.
Abstract The scope of this study was to identify the levels of satisfaction of the elderly regarding the organization of services in primary health care services. It involved a cross-sectional survey of 18,671 users of the Unified Health System. Information on satisfaction with care was collected, categorized as satisfied (very good, good and average perception) and dissatisfied (poor and very poor). The aspects researched included: characteristics of the organization of care and the secondary data from the 1st cycle of the Brazilian Program for Improving Access and Quality in Primary Health Care (PMAQ-AB). Logistic regression was used to obtain the odds ratio (OR) and confidence interval (CI95\%), adjusted for sociodemographic factors. The prevalence of satisfaction was $87 \%$. The aspects associated with satisfaction $(p<0.05)$, after analysis of factors that could distort the results, were access to the health unit, home visit and availability of medication - related to the organization of services; and, performing an active search, solving the problem in the unit, as well as the physical examination and consultation time - related to the organization of care. The analyses describe multiple aspects of the organization of care and services associated with the satisfaction of the elderly with basic health care services.

Key words User satisfaction, Primary Health Care, Elderly
Resumo Este estudo objetivou identificar os preditores da satisfação dos idosos em relação à organização dos serviços e do cuidado na atenção básica à saúde. Delineamento seccional, entre 18.671 usuários da Atenção Básica do Sistema Único de Saúde. Foram coletadas informações sobre a satisfação com o cuidado, categorizada em satisfeito (percepção muito boa, boa e regular) e insatisfeito (ruim e muito ruim). As exposições pesquisadas incluíram: características da organização do serviço e do cuidado, a partir de dados secundários do $1^{\circ}$ ciclo do PMAQ-AB. Foi utilizada regressão logística para obter os odds ratio (OR) e os respectivos intervalos de confiança (95\%), ajustado por fatores sociodemográficos. A prevalência de satisfação foi de $87,0 \%$. Os preditores associados $(p<0,05)$ à satisfação, após análise por fatores que poderiam confundir os resultados, foram acesso à unidade de saúde, visita domiciliar e disponibilidade de medicamento - relacionados à organização dos serviços; e, realização de busca ativa, resolução do problema na unidade, assim como ao exame físico realizado e tempo da consulta - relacionados à organização do cuidado. Em sintese, as análises descrevem múltiplos preditores da organização do cuidado e dos serviços associados à satisfação dos idosos com os serviços da atenção básica.

Palavras-chave Satisfação do usuário, Atenção Primária à Saúde, Idoso 


\section{Introdução}

O crescente contingente de idosos acarreta um acréscimo das condições crônicas, múltiplas, não transmissíveis próprias do envelhecimento e problemas associados, aumentando a procura por serviços em todos os níveis de complexidade ${ }^{1}$, especialmente na atenção básica. Embora as intervenções neste nível de densidade tecnológica dos serviços sejam capazes de melhorar a qualidade de vida e diminuir a necessidade futura de formas de tratamento mais dispendiosas e traumáticas entre idosos ${ }^{2}$, no Brasil ainda são escassas e recentes as evidências que analisam os aspectos da oferta de serviços relacionados à organização do trabalho ou do cuidado prestado. A prática de avaliar a qualidade dos serviços de saúde por meio da satisfação dos usuários difundiu-se em toda America Latina, inicialmente com foco na adesão ao tratamento, principalmente como parte importante dos processos de planejamento e avaliação $0^{3,4}$.

A avaliação da satisfação comporta sempre duas dimensões: desempenho técnico, ou seja, aplicação do conhecimento e da tecnologia médica, de modo a maximizar os benefícios e reduzir os riscos; e, relacionamento com o paciente ${ }^{5-7}$. A avaliação do cuidado à saúde pode ser, então, realizada a partir dos componentes de estrutura (características dos recursos utilizados pelo serviço), do processo (procedimentos empregados no manejo dos problemas apresentados pelos pacientes) e do resultado (estado de saúde do paciente ou da comunidade resultante da interação com o serviço de saúde). Assim, a qualidade do cuidado é uma importante ferramenta no planejamento e na gestão dos serviços e sistemas de saúde, é multifatorial e a satisfação dos usuários é um marcador relevante $e^{3,6,8}$.

Tempo de espera, acompanhamento longitudinal, capacidade resolutiva do serviço de saúde, respeito aos sentimentos e crenças pelos profissionais, horário de funcionamento, escuta qualificada, espaço para interação entre profissional e usuário e realização de visita domiciliar são os fatores determinantes da satisfação dos usuários ${ }^{9-11}$. Contudo, esses resultados decorrem de grupos populacionais que nem sempre incluem idosos, os quais têm especificidades relativas ao modo de vida, em utilizar e acessar aos serviços de saúde. Assim, no âmbito da organização dos serviços da atenção básica, a expansão relevante nos últimos anos, principalmente após a implementação do Sistema Único de Saúde (SUS), e a adesão ao Programa de Melhoria do Acesso e Qualidade da
Atenção Básica (PMAQ-AB) tem envolvido um expressivo volume de serviços e usuários ${ }^{12}$.

Visando melhorar a qualidade dos serviços e a cobertura populacional a partir da Estratégia Saúde da Família foi a implantada pelo Ministério da Saúde a iniciativa de avaliação da atenção básica por meio da adesão dos municípios e equipes ao PMAQ-AB, pela qual são repassados recursos adicionais com fontes de financiamento federal para os municípios participantes que atingem melhores indicadores no padrão de qualidade e de acesso no atendimento, por meio de avaliação periódica sobre o próprio trabalho dos profissionais, os indicadores de saúde e padrões de qualidade, este último avaliado por equipe externa e cidadãos. As ações territoriais de assistência, vigilância e prevenção, com populações adscritas aos serviços são prioridades, e idosos estão incluídos entre os grupos populacionais prioritários $^{13}$. A satisfação do usuário idoso está associada à utilização do serviço, adesão à proposta terapêutica e manutenção de vínculo com o prestador do atendimento ${ }^{3,9-11,13}$, contudo, a análise dessa questão em maior profundidade ainda requer compreensão da superação das possíveis deficiências de infraestrutura ainda existentes no sistema de saúde que podem, conforme propõe Donabedian ${ }^{5}$, influenciar negativamente a avaliação dos serviços pelos usuários. Assim, o presente estudo visa avaliar a satisfação de usuários idosos com os serviços da atenção básica e identificar preditores da organização do cuidado e dos serviços em municípios brasileiros que aderiram a proposta do PMAQ-AB.

\section{Métodos}

Foi realizado um estudo baseado em dados secundários do $1^{\circ}$ ciclo do Programa Nacional de Melhoria do Acesso e da Qualidade da Atenção Básica no Brasil (PMAQ-AB), o qual tem delineamento transversal, exploratório, de natureza avaliativa, teve abrangência nacional e foi executado de forma multicêntrica e integrada, por instituições de Ensino e Pesquisa e com o acompanhamento direto do Ministério da Saúde do Brasil. A presente pesquisa possui como base o projeto de pesquisa guarda-chuva intitulado Avaliação da atenção básica no Brasil: estudos multicêntricos integrados sobre acesso, qualidade e satisfação dos usuários, aprovadas pelo Comitê de Ética em Pesquisa da Universidade Federal do Rio Grande do Sul.

Participaram do estudo 17.203 Equipes de Saúde da Atenção Básica do Sistema Único de 
Saúde (SUS) que aderiram à avaliação, localizadas em 3.944 municípios brasileiros. Entre os serviços de saúde participantes do PMAQ-AB, $63,7 \%$ enquadram-se na modalidade de Centro de Saúde/Unidade de Saúde, 26,9\% como Posto de Saúde e 9,3\% como Postos Avançados/Outros. Mais especificamente, analisaram-se as respostas de 65.392 usuários entrevistados no Brasil no Módulo III - Entrevista na Unidade de Saúde com Usuário, que objetivou averiguar a satisfação destes quanto aos serviços de saúde em relação ao seu acesso e utilização. Contudo, para o recorte da avaliação com idosos considerou-se dados completos dos indivíduos de 60 anos e mais e variáveis com $100 \%$ de dados presentes. Assim, o estudo considerou 18.671 idosos, grupo que representa $69,81 \%$ dos 26.746 idosos usuários entrevistados.

Desse modo, o presente estudo caracteriza as variáveis em níveis individual, de organização do cuidado e do serviço. No nível individual, o qual diz respeito aos idosos, foram elencadas questões referentes às características sociodemográficas, utilizando como variáveis a idade, sexo, cor autodeclarada e escolaridade, a partir da entrevista com os idosos usuários (Módulo III). No bloco sobre a satisfação com o cuidado, a partir da pergunta norteadora sobre a percepção de satisfação com o cuidado recebido da equipe de saúde, foi categorizada em satisfeito e insatisfeito. A partir de uma escala likert, foram considerados satisfeitos aqueles idosos que referiram a percepção como muito boa, boa e regular e como insatisfeitos aqueles que relataram ruim e muito ruim. A avaliação da satisfação do cuidado foi realizada quanto ao acesso e utilização por meio de um bloco de questões sobre disponibilidade de medicamentos, possibilidade de mudança de equipe, recomendação do serviço, o cuidado pessoal e familiar e percepção de cuidado recebido por profissionais. Estas variáveis foram consideradas dentro de um modelo teórico apresentado na Figura 1.

Considerou-se como variáveis independentes as informações respondidas no Módulo III (entrevista com usuário) sobre os preditores relacionados à 1) organização do cuidado: busca ativa dos idosos pelos profissionais, interesse dos profissionais em resolver os problemas no próprio serviço, realização de exame físico (se necessário) e satisfação com o tempo de consulta; e, 2) organização com os serviços: facilidade de acesso ao serviço, funcionamento em horário integral, visita domiciliar por Agente Comunitário de Saúde, disponibilidade do medicamento prescrito e facilidade na marcação de consulta por telefone.

Para a análise dos dados foi feita a correção pelo efeito desenho por ponderação, assim, a análise descritiva incluiu frequência absoluta (n) sem correção e frequência relativa (\%) com correção para variáveis categóricas, média e desvio padrão (DP), para as contínuas. Para análise dos dados foi adotada estatística bivariada utilizando-se o teste do qui quadrado de Pearson e ANOVA.

Todas as variáveis consideradas neste trabalho foram incluídas, uma a uma, nos modelos logísticos iniciais; aquelas que permaneceram associadas com a variável dependente em nível inferior a 0,05 foram mantidas no modelo final, ajustadas por idade, sexo, escolaridade, cor autodeclarada e região geográfica de moradia do participante. A regressão logística binária e a correção pelo efeito de delineamento do estudo foram utilizadas para estimar odds ratio (OR) bruta e ajustada nas análises univariadas e multivariada de associações da variável dependente com as variáveis explicativas e os intervalos de $95 \%$ de confiança (IC95\%) com correção pelo efeito de desenho. $\mathrm{O}$ teste de Hosmer-Lemeshow foi utilizado para verificar a adequação do modelo final. As análises foram realizadas utilizando-se os procedimentos do programa Stata versão 13.0 (Stata Corporation, College Station, Texas) para inquéritos populacionais.

\section{Resultados}

A satisfação com serviços e cuidado na atenção básica foi de 87,0\% (IC95\%: 86,5-87,4) dos usuários. A média de idade geral dos participantes foi de 68,5 anos $( \pm 6,5)$, entre os insatisfeitos foi de $67,7( \pm 6,0), 64,4 \%$ eram mulheres, $5,9 \%$ declararam não saber ler e escrever e $44,6 \%$ eram brancos. Todas as variáveis selecionadas para compor a análise apresentaram diferença estatisticamente significativa em relação à percepção do cuidado. A Tabela 1 apresenta as características sociodemográficas, de organização do serviço e organização do cuidado, conforme a percepção de satisfação dos idosos brasileiros de 60 anos ou mais participantes PMAQ-AB.

$\mathrm{Na}$ Tabela 2, são apresentados os resultados da análise bruta e ajustada da associação entre variáveis relacionadas à organização dos serviços e o relato da percepção de satisfação dos idosos. Quando o acesso ao serviço era razoável (OR: 1,83; IC95\%: 1,62-2,06) ou muito difícil (OR: 2,13; IC95\%: 1,88-2,42), assim como quando ha- 


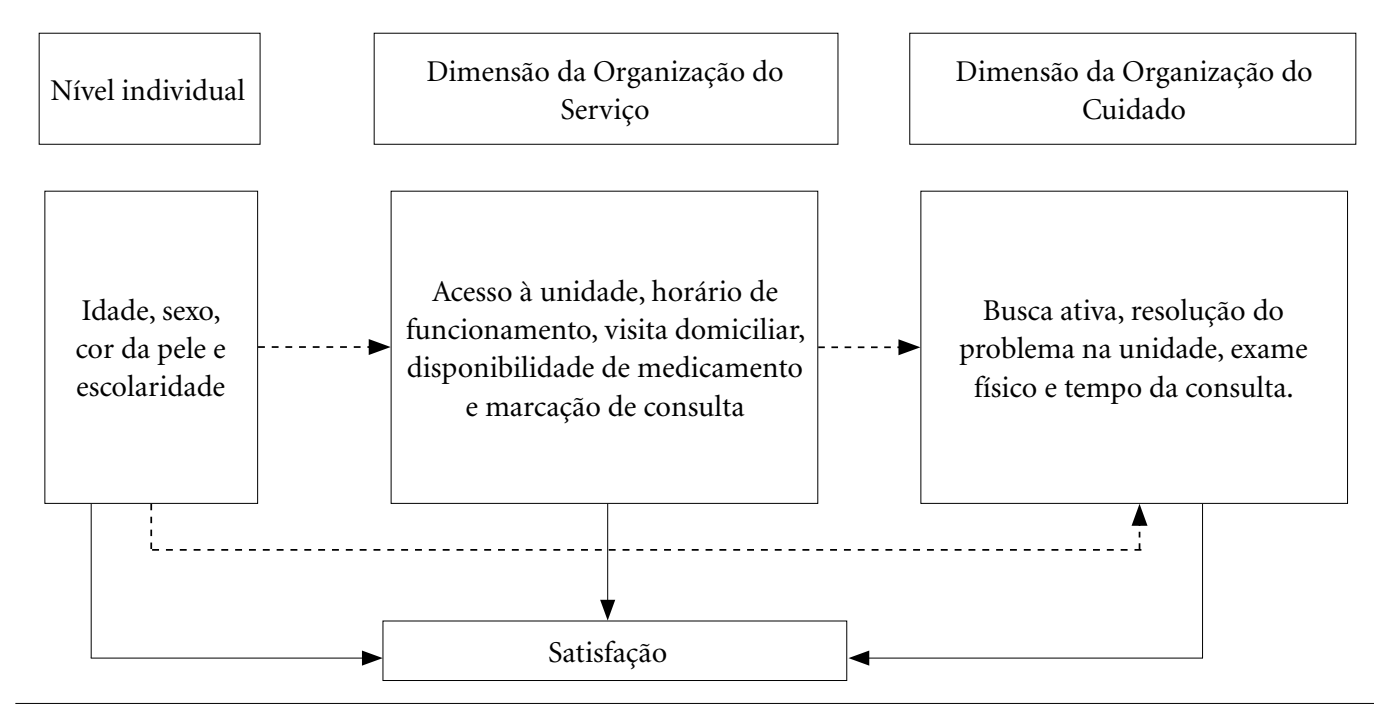

Figura 1. Modelo conceitual de fatores associados à satisfação do idoso usuário do PMAQ-AB.

via disponibilidade de medicamento na maioria das vezes (OR: 1,73; IC95\%: 1,50-1,98), quase nunca (OR: 4,42; IC95\%: 3,83-5,09) ou nunca (OR: 4,52; IC95\%: 3,80-5,37), observou-se associação à maior chance de perceber satisfação na atenção básica. Quando o horário de funcionamento do serviço não era em tempo integral (manhã e tarde) houve $30 \%$ a mais na chance de obter uma avaliação ruim, mas não no modelo ajustado. Disponibilidade de marcação de consulta por telefone também contribuiu com as chances de satisfação (OR: 0,72; IC95\%: 0,590,88).

As características da organização do cuidado também foram analisadas na perspectiva da satisfação dos idosos. Os resultados apresentados na Tabela 3 são da análise bruta e ajustada referentes a associação entre as características da organização do cuidado e a percepção de satisfação do idoso. Considerando os ajustes por características do próprio bloco, observou-se um efeito gradiente na associação da satisfação com busca ativa quase sempre ou na maioria das vezes (OR: 0,63; IC95\%: 0,55-0,72) e quase nunca ou nunca (OR: 3,00; IC95\%: 2,72-3,32), quando o problema de saúde era resolvido na própria unidade na maioria das vezes (OR: 2,54; IC95\%: 2,29-2,81), quase nunca (OR: 9,80; IC95\%: 8,49-11,30) ou nunca (OR: 16,80; IC95\%: 13,44-21,00), mas também quando o exame físico era realizado na maioria das vezes (OR: 1,73; IC95\%: 1,51-1,98), quase nunca (OR: 3,73; IC95\%: 3,26-4,27) e nunca (OR: 4,22; IC95\%: 3,77-4,73). A satisfação com o tempo da consulta também mostrou associação significativa com a satisfação, porém de modo inverso (OR: 0,22; IC95\%: 0,20-0,24).

Os resultados finais da análise múltipla dos fatores associados à organização dos serviços e do cuidado e satisfação do idoso estão apresentados na Tabela 4. Observa-se que a satisfação quanto à organização dos serviços esteve associada o acesso razoável (OR: 1,50; IC95\%: 1,32-1,71) ou muito difícil (OR: 1,47; IC95\%: 1,28-1,70), nunca realizar busca ativa (OR: 1,81; IC95\%: $1,62-2,02)$ e quando na maioria das vezes (OR: 1,38; IC95\%: 1,19-1,60), quase nunca (OR: 2,40; IC95\%: 2,06-2,80) ou nunca (OR: 2,14; IC95\%: 1,77-2,59) havia disponibilidade de medicamento. A satisfação também foi associada aos preditores da organização do cuidado no que se refere a resolução do problema na unidade na maioria das vezes (OR: 1,89; IC95\%: 1,70-2,10), quase nunca (OR: 4,67; IC95\%: 4,00-5,45) ou nunca (OR: 7,21; IC95\%: 5,65-9,21), assim como ao exame físico realizado na maioria das vezes (OR: 1,28; IC95\%: 1,11-1,48), quase nunca (OR: 1,98; IC95\%: 1,71-2,30) ou nunca (OR: 1,99; IC95\%: 1,76-2,62). A realização de visita domiciliar e satisfação com o tempo da consulta diminuem 1,56 (IC95\%: 0,56-0,72) e 2,22 vezes (IC95\%: 0,40$0,51)$ as chances de satisfação. 
Tabela 1. Características sociodemográficas, organização do serviço e organização do cuidado, de acordo com a percepção de satisfação dos idosos brasileiros de 60 anos ou mais. Módulo III do $1^{\circ}$ Ciclo do Programa Nacional de Melhoria do Acesso e da Qualidade da Atenção Básica no Brasil (PMAQ-AB), 2013.

\begin{tabular}{|c|c|c|c|c|}
\hline \multirow[b]{2}{*}{ Variáveis } & \multirow[b]{2}{*}{$\begin{array}{c}\text { Total } \\
(\mathbf{n}=\mathbf{1 8 . 6 7 1})\end{array}$} & \multicolumn{2}{|c|}{ Percepção com cuidado } & \multirow[b]{2}{*}{ p-valor } \\
\hline & & $\begin{array}{c}\text { Satisfeito } \\
16.236 \\
(87,0 \%)\end{array}$ & $\begin{array}{c}\text { Insatisfeito } \\
2.435 \\
(13,0 \%)\end{array}$ & \\
\hline \multicolumn{5}{|l|}{ Organização do Serviço } \\
\hline Acesso à unidade, $\%$ & & & & $<0,001$ \\
\hline Muito fácil & 78,1 & 79,8 & 67,0 & \\
\hline Razoável & 12,4 & 11,6 & 17,7 & \\
\hline Muito difícil & 9,5 & 8,7 & 15,3 & \\
\hline Funcionamento em horário integral, \% & 95,4 & 95,6 & 94,4 & $<0,001$ \\
\hline Visita domiciliar por ACS, $\%$ & 87,7 & 89,4 & 76,0 & $<0,001$ \\
\hline Disponibilidade medicamento, $\%$ & & & & $<0,001$ \\
\hline Sempre & 24,6 & 26,3 & 12,8 & \\
\hline Maioria das vezes & 38,3 & 39,1 & 32,9 & \\
\hline Quase nunca & 16,7 & 14,4 & 31,7 & \\
\hline Nunca & 6,9 & 5,9 & 13,3 & \\
\hline Não dispensado & 13,6 & 14,2 & 9,4 & \\
\hline Marcação de consulta por telefone, \% & 96,1 & 96,3 & 94,8 & $<0,001$ \\
\hline \multicolumn{5}{|l|}{ Organização do Cuidado } \\
\hline Busca ativa, $\%$ & & & & $<0,001$ \\
\hline Nunca interrompeu o tratamento & 64,2 & 65,6 & 54,5 & \\
\hline Quase sempre ou maioria & 19,5 & 20,8 & 11,1 & \\
\hline Quase nunca ou nunca & 15,6 & 13,0 & 33,5 & \\
\hline \multicolumn{5}{|l|}{ Resolução do problema na unidade, \% } \\
\hline Sempre & 57,7 & 61,7 & 30,6 & $<0,001$ \\
\hline Maioria das vezes & 34,6 & 33,4 & 42,1 & \\
\hline Quase nunca & 5,8 & 3,9 & 19,1 & \\
\hline Nunca & 1,9 & 1,0 & 8,2 & \\
\hline Exame físico, \% & & & & $<0,001$ \\
\hline Sempre & 45,3 & 48,7 & 23,0 & \\
\hline Maioria das vezes & 19,9 & 20,4 & 16,7 & \\
\hline Quase nunca & 12,1 & 11,0 & 19,2 & \\
\hline Nunca & 22,8 & 20,0 & 41,2 & \\
\hline Satisfação com tempo da consulta, \% & 88,7 & 91,5 & 69,9 & $<0,001$ \\
\hline
\end{tabular}

\section{Discussão}

O presente estudo evidenciou que, apesar da maioria dos participantes relatar satisfação com os serviços de saúde da atenção básica que aderiram ao PMAQ (87,0\%), ainda se observa uma prevalência considerável de idosos que apresentam insatisfação com a organização do cuidado e dos serviços. Este achado está em consonância

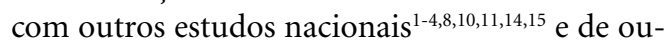
tros países ${ }^{9,16-20}$.

Em relação aos fatores associados, os preditores da percepção de satisfação modelados nes- 
Tabela 2. Associação entre o relato de percepção de satisfação dos idosos e variáveis de organização do serviço. Módulo III do $1^{\circ}$ Ciclo do Programa Nacional de Melhoria do Acesso e da Qualidade da Atenção Básica no Brasil (PMAQ-AB), 2013.

\begin{tabular}{|c|c|c|}
\hline \multirow{2}{*}{ Variáveis } & \multicolumn{2}{|c|}{ Odds Ratio (Intervalo de Confiança de 95\%) } \\
\hline & Bruta & Ajustada \\
\hline \multicolumn{3}{|l|}{ Acesso à unidade } \\
\hline Muito fácil & 1,00 & 1,00 \\
\hline Razoável & $1,83(1,63-2,05)$ & $1,83(1,62-2,06)$ \\
\hline Muito difícil & $2,10(1,85-2,38)$ & $2,13(1,88-2,42)$ \\
\hline Funcionamento em horário integral (sim) & $0,78(0,65-0,94)$ & $0,89(0,74-1,09)$ \\
\hline Visita domiciliar por ACS (sim) & $0,37(0,34-0,42)$ & $0,37(0,33-0,42)$ \\
\hline \multicolumn{3}{|l|}{ Disponibilidade medicamento } \\
\hline Sempre & 1,00 & 1,00 \\
\hline Maioria das vezes & $1,76(1,51-1,98)$ & $1,73(1,50-1,98)$ \\
\hline Quase nunca & $4,51(3,92-5,19)$ & $4,42(3,83-5,09)$ \\
\hline Nunca & $4,63(3,90-5,49)$ & $4,52(3,80-5,37)$ \\
\hline Marcação de consulta por telefone (sim) & $0,70(0,57-0,85)$ & $0,72(0,59-0,88)$ \\
\hline
\end{tabular}

Modelo ajustado: características sociodemográficas, região geográfica de moradia; R2 corrigido: 8,1\%; Likelihood ratio test (LR teste) $=1162,11 ; \mathrm{p}<0,000)$.

chances de obter a satisfação dos usuários com os serviços. Logo, os principais motivos associados à satisfação dos usuários são: a boa localização do serviço, a pouca distância da unidade até seu domicílio, o curto intervalo de tempo gasto no percurso até a unidade e a possibilidade de não utilizar meio de transporte ${ }^{14}$. Espera-se que os obstáculos relacionados à distância estejam associados aos níveis secundários de atenção, pois os serviços da atenção básica devem estar localizados próximos à residência dos usuários ${ }^{21}$. Em se tratando de idosos, a reflexão acerca da deficiência na mobilidade e na acessibilidade podem ser limitadores da utilização plena uma vez que a tarefa de projetar ambientes para estes usuários ainda é tratada de forma bastante superficial ${ }^{22}$.

Na dimensão da organização dos serviços, os resultados da pesquisa que se descreve nesse artigo apontam a visita domiciliar realizada pelos agentes comunitários de saúde como fator influenciador da satisfação, o que corrobora outros estudos $^{11,15,21}$. No contexto da $\mathrm{AB}$, a atenção domiciliar é uma grande oportunidade para acesso dos idosos aos serviços de saúde por meio da capilarização da oferta de serviços para chegar o mais próximo do usuário, porta da interface de diálogo entre profissionais de saúde e o indivíduo sob cuidado em casos de limitações físicas e incapacidade estabelecidas e uma via para a garantia de direitos como integralidade, humanização, acesso e cuidado centrado no usuário e sua família ${ }^{21}$. Analisando-se a prática de busca ativa pelos profissionais, os usuários idosos compreenderam-na como interesse da equipe em conhecer antecipadamente as necessidades de saúde da sua população, elevando-se assim os níveis da satisfação. Visto que a população ainda tem uma educação voltada para a lógica curativista, a ação dos profissionais no sentido contrário do automatismo da demanda espontânea e da queixa-conduta é vista como um princípio organizador dos processos de trabalho nas atuais políticas de saúde e da atenção básica, na construção de práticas de cuidado pautadas por uma lógica territorial e, desta forma, é relevante transpor as ações de saúde para mais perto do mundo do usuário e de seus modos de vida. Isso permite que orientações e intervenções no ambiente físico do próprio idoso, o planejamento de atividades, a prevenção de agravos e o monitoramento das condições de saúde potencialize a atenção básica. Buscar soluções alternativas para as necessidades apresentadas pelos idosos dentro da própria unidade reduz os encaminhamentos e, consequentemente, as filas de espera ou agravamento da condição de saúde $^{14}$. Como se trata de um grupo populacional onde o deslocamento é condição sensível, a resolutividade local também reduz o dispêndio de recursos para acesso a outros serviços. Nesse sentido, é possível que esse aspecto seja resolvido com uma abordagem acolhedora, valorizando as queixas e buscando possíveis soluções para o problema do usuário mesmo que seja necessário a referência para outros serviços ${ }^{23}$. Esse resultado 
Tabela 3. Características da organização do cuidado associadas ao relato de percepção de satisfação dos idosos. Módulo III do $1^{\circ}$ Ciclo do Programa Nacional de Melhoria do Acesso e da Qualidade da Atenção Básica no Brasil (PMAQ-AB), 2013.

\begin{tabular}{|c|c|c|}
\hline \multirow{2}{*}{ Variáveis } & \multicolumn{2}{|c|}{ Odds ratio (Intervalo de Confiança 95\%) } \\
\hline & Bruta & Ajustada \\
\hline \multicolumn{3}{|l|}{ Busca ativa dos idosos } \\
\hline Nunca interrompeu o tratamento & 1,00 & 1,00 \\
\hline Quase sempre ou maioria & $0,64(0,56-0,74)$ & $0,63(0,55-0,72)$ \\
\hline Quase nunca ou nunca & $3,11(2,82-3,43)$ & $3,00(2,72-3,32)$ \\
\hline \multicolumn{3}{|l|}{ Resolução do problema na unidade } \\
\hline Sempre & 1,00 & 1,00 \\
\hline Maioria das vezes & $2,54(2,30-2,81)$ & $2,54(2,29-2,81)$ \\
\hline Quase nunca & $9,95(8,64-11,45)$ & $9,80(8,49-11,30)$ \\
\hline Nunca & $16,94(13,58-21,13)$ & $16,80(13,44-21,00)$ \\
\hline \multicolumn{3}{|l|}{ Exame físico } \\
\hline Sempre & 1,00 & 1,00 \\
\hline Maioria das vezes & $1,74(1,52-1,99)$ & $1,73(1,51-1,98)$ \\
\hline Quase nunca & $3,71(3,24-4,23)$ & $3,73(3,26-4,27)$ \\
\hline Nunca & $4,37(3,91-4,88)$ & $4,22(3,77-4,73)$ \\
\hline Satisfação com tempo da consulta & $0,22(0,19-0,24)$ & $0,22(0,20-0,24)$ \\
\hline
\end{tabular}

Modelo bruto sem ajuste. Modelo ajustado: características sociodemográficas, região geográfica de moradia; R2 corrigido: 16,4\%; Likelihood ratio test $($ LR teste $)=2355,7 ; \mathrm{p}<0,000)$.

corrobora com estudo de Bernhart et al. ${ }^{9}$, realizado na Indonésia, que encontrou, como principal aspecto para obter a satisfação do serviço, o fato de o usuário sentir que o seu problema foi resolvido.

As associações clássicas entre a satisfação com o serviço e o funcionamento foram replicadas neste estudo. Esse resultado reforça estudo anterior $^{14}$, contudo, observa-se que em adultos o horário pode prejudicar a acessibilidade do serviço devido às suas atividades ocupacionais em horário comercial, mas para idosos essa justificativa não parece completamente suficiente, na medida em que a vinculação ao trabalho formal é menos frequente nessa faixa da população. Parece que o horário de funcionamento está vinculado ao modelo restritivo de acesso, com formação de filas de espera e prática de distribuição de senha, o que em muitos casos remete ao modelo não universal de acesso $^{14,15,23,24}$.

Para além das tecnologias leves, de natureza relacional, que envolvem a produção de vínculo e o acolhimento no serviço, a disponibilidade de medicamento e realização de exame físico completo, neste estudo, foram determinantes importantes na compreensão da satisfação do usuário idoso. Quanto maior a frequência de disponibilidade de medicamentos na unidade de saúde, melhor foi a satisfação do usuário avaliado. Quando os esto- ques encontram preenchidos em suas farmácias, a probabilidade foi $26,7 \%$ de maior satisfação, o que corrobora outros estudos ${ }^{25,26}$. O processo de acesso aos medicamentos essenciais pode favorecer maior adesão à terapia e menor quantidade de estoque domiciliar. No caso da população idosa, mais frequentemente acometida por doenças crônicas e com uso regular de medicamentos, a importância da disponibilidade desse insumo na avaliação sobre a qualidade da atenção recebida nos serviços de saúde é facilmente compreendida.

Embora alguns autores já tivessem sugeridos a relevância do exame físico no idoso, os nossos resultados reforçam esta evidência na propedêutica e para a prescrição terapêutica ${ }^{20,27,28}$. O exame deve ir além da avaliação clínica (anamnese), pois está diretamente associado à sensação de cuidado individual e completo. No caso dos mais longevos, em que são frequentes declínios das capacidades vitais e maior prevalência de doenças e agravos, o exame físico é muito relevante. Esse domínio se refere à relação entre usuário e profissional, ou seja, o atendimento cuidadoso, o interesse pelo seu estado de saúde, o toque, a ausculta, a percussão, quando necessários, são algumas das informações pertinentes para o usuário demonstrar a satisfação com o serviço.

Observou-se em nossos resultados que quando não houve satisfação com o tempo da con- 
Tabela 4. Modelo final da regressão logística dos preditores associados à satisfação, conforme a organização do serviço e organização do cuidado. Módulo III do $1^{\circ}$ Ciclo do Programa Nacional de Melhoria do Acesso e da Qualidade da Atenção Básica no Brasil (PMAQ-AB), 2013.

\begin{tabular}{|c|c|c|}
\hline \multirow[b]{2}{*}{ Variáveis } & \multicolumn{2}{|c|}{ Análise Multivariada } \\
\hline & $\begin{array}{l}\text { Odds } \\
\text { Ratio }\end{array}$ & $\begin{array}{c}\text { Intervalo } \\
\text { de } \\
\text { Confiança } \\
\text { de } 95 \%\end{array}$ \\
\hline \multicolumn{3}{|l|}{ Acesso à unidade } \\
\hline Muito fácil & 1,00 & - \\
\hline Razoável & 1,50 & $1,32-1,71$ \\
\hline Muito difícil & 1,47 & $1,28-1,70$ \\
\hline \multicolumn{3}{|l|}{ Busca ativa dos idosos } \\
\hline $\begin{array}{l}\text { Nunca interrompeu o } \\
\text { tratamento }\end{array}$ & 1,00 & - \\
\hline $\begin{array}{l}\text { Quase sempre ou } \\
\text { maioria }\end{array}$ & 0,88 & $0,76-1,01$ \\
\hline Quase nunca ou nunca & 1,81 & $1,62-2,02$ \\
\hline $\begin{array}{l}\text { Visita domiciliar por } \\
\text { ACS (sim) }\end{array}$ & 0,64 & $0,56-0,72$ \\
\hline \multicolumn{3}{|l|}{$\begin{array}{l}\text { Disponibilidade } \\
\text { medicamento }\end{array}$} \\
\hline Sempre & 1,00 & - \\
\hline Maioria das vezes & 1,38 & $1,19-1,60$ \\
\hline Quase nunca & 2,40 & $2,06-2,80$ \\
\hline Nunca & 2,14 & $1,77-2,59$ \\
\hline $\begin{array}{l}\text { Marcação de consulta } \\
\text { por telefone (sim) }\end{array}$ & 0,87 & $0,70-1,09$ \\
\hline \multicolumn{3}{|l|}{$\begin{array}{l}\text { Resolução do problema na } \\
\text { unidade }\end{array}$} \\
\hline Sempre & 1,00 & - \\
\hline Maioria das vezes & 1,89 & $1,70-2,10$ \\
\hline Quase nunca & 4,67 & $4,00-5,45$ \\
\hline Nunca & 7,21 & $5,65-9,21$ \\
\hline \multicolumn{3}{|l|}{ Exame físico } \\
\hline Sempre & 1,00 & - \\
\hline Maioria das vezes & 1,28 & $1,11-1,48$ \\
\hline Quase nunca & 1,98 & $1,71-2,30$ \\
\hline Nunca & 1,99 & $1,76-2,62$ \\
\hline $\begin{array}{l}\text { Satisfação com tempo da } \\
\text { consulta }\end{array}$ & 0,45 & $0,40-0,51$ \\
\hline
\end{tabular}

Modelo ajustado: características sociodemográficas, região geográfica de moradia e variáveis das dimensões de organização do serviço e cuidado; R2 corrigido: $18,1 \%$; Likelihood ratio test $($ LR teste $)=2603,1 ; \mathrm{p}<0,000)$.'

sulta, a percepção da satisfação com o serviço foi limitada. O tempo de consulta é interpretado pelos usuários com a realização de exame físico completo, espaço para escuta qualificada, discus- são do plano terapêutico, reavaliação de condições previamente apresentadas, o encadeamento informacional ou no esclarecimento de dúvidas. Do ponto de vista da saúde pública, para os idosos, especialmente, esses determinantes implicam fortemente na satisfação com a atenção recebida e na percepção de cuidado singular, uma vez que o maior tempo de contato com os profissionais e serviços vai ao encontro da qualidade do atendimento, pois possibilita uma melhor anamnese, uma melhor apreciação do problema e dos procedimentos diagnósticos e terapêuticos, assim como a verificação do médico sobre a compreensão do paciente e a participação do paciente na consulta 27,29 .

No que se refere às limitações deste estudo, embora o desenho transversal tenha sido adequado à investigação dos objetivos estabelecidos (estimar a satisfação de usuários idosos com a qualidade dos serviços da atenção básica no Brasil, e os fatores a eles associados), esse delineamento não permite estabelecer relações temporais entre a satisfação e as variáveis independentes, podendo algumas dessas associações serem resultado de causalidade reversa. Outra limitação a ser considerada é a possibilidade de viés de informação e de seleção, por omissão de dados, sobretudo no que se refere à insatisfação, e por não se tratar de amostra probabilística, o que poderia ter reduzido a magnitude das associações reportadas. Por outro lado, trata-se de um estudo conduzido em uma amostra nacional, que se utilizou de procedimentos padronizados para a coleta das informações, assegurando sua validade interna ${ }^{12}$. Também outros domínios precisam ser investigados, pois para melhor compreensão dos resultados encontrados há necessidade de identificar e analisar fatores associados, não considerados nesta pesquisa, a exemplo das diferenças culturais, comportamentais e condições de saúde que afetam a satisfação do usuário ${ }^{17,18}$. Sugerimos novos delineamentos com abordagem qualitativa, uma vez que a investigação quantitativa fornece indícios sobre qualidade em serviços, mas que, sobretudo no que tange à percepção de usuários, precisam ser qualitativamente ponderados.

Em síntese, os preditores da organização dos serviços e do cuidado na atenção básica no Sistema Único de Saúde associados à satisfação dos idosos foram múltiplos, mas todos modificáveis. Incorporar a avaliação da satisfação do usuário, especialmente idoso, é, sobretudo, delinear estratégias, critérios e padrões de medição, a fim de auxiliar na tomada de decisão e subsidiar aperfeiçoamentos no âmbito dos serviços. Esses re- 
sultados são importantes para a compreensão da assistência à saúde dos idosos, para compreender as realidades múltiplas e diversas que compõem a organização dos serviços, mas também para colaborar no direcionamento de políticas que visam a melhoria do acesso e da qualidade da atenção básica e tornar-se como meta a ser alcançada pelos serviços que atendam os idosos. Atender as expectativas dos idosos pode ser uma estratégia de melhorar a adesão deles aos tratamentos prescritos, a continuarem utilizando os serviços de saúde, contribuir para uma melhor qualidade de vida.

\section{Colaboradores}

JSC Amorim participou da concepção, planejamento, análise, interpretação e redação do trabalho. LB Teixeira participou no planejamento e interpretação do trabalho. AA Ferla participou da concepção, planejamento, interpretação e redação do trabalho. Todos os autores aprovaram a versão final encaminhada.

\section{Agradecimentos}

Ao Conselho Nacional de Desenvolvimento Científico e Tecnológico (CNPq), e à Fundação de Amparo à Pesquisa do Estado do Rio Grande do Sul (FAPERGS) pelo financiamento do presente trabalho. 


\section{Referências}

1. Almeida AN. The access to health services by elderly people in Brazil based on National Household Sample Survey (PNAD) from 1998 to 2008. J Bras Econ Saúde 2015; 7(1):43-52.

2. Paskulin LMG, Valer DB, Vianna LAC. Use and access of the elderly to primary health care services in Porto Alegre (RS, Brasil). Cien Saude Colet 2011; 16(6):2935-2944.

3. Trad LAB, Bastos ACS, Santana EM, Nunes MO. Estudo etnográfico da satisfação do usuário do Programa de Saúde da Família (PSF) na Bahia. Cien Saude Colet 2002; 7(3):581-589.

4. Paes NA, Silva CS, Figueiredo TMRM, Cardoso MAA, Lima JO. Satisfação dos usuários hipertensos com os serviços da rede de atenção primária no Brasil: um estudo de validação. Rev Panam Salud Publica 2014; 36(2):87-93.

5. Donabedian A. The definition of quality: a conceptual exploration. Explorations is quality assessment and monitoring. In: the definition of quality and approaches to its assessment. Ann Arbor: Health Administration Press; 1988.

6. Esperidião MA, Trad LAB. Avaliação de satisfação de usuários: considerações teórico-conceituais. Cad Saude Publica 2006; 22(6):1267-1276.

7. Pinheiro R, Martins PH, organizadores. Avaliação em saúde na perspectiva do usuário: abordagem multicêntrica. Rio de Janeiro: CEPESC/IMS-UERJ, Recife: Editora Universitária UFPE, São Paulo: ABRASCO; 2009.

8. Bastos GAN, Fasolo LR, Bastos GAN, Fasolo LR. Factors that influence outpatient service user satisfaction in a low-income population: a population-based study. Rev Bras Epidemiol 2013; 16(1):114-124.

9. Bernhart MH, Wiadnyana IGP, Wihardjo H, Pohan I. Patient satisfaction in developing countries. Soc Sci Med 1999; 48(8):989-996.

10. Protasio APL, Gomes LB, Machado LS, Valença AMG. Satisfação do usuário da Atenção Básica em Saúde por regiões do Brasil: $1^{\circ}$ ciclo de avaliação externa do PMAQ-AB. Cien Saude Colet 2017; 22(6):18-29.

11. Mues KE, Resende JC, Santos OC, Perez LG, Ferreira JA, Leon JS. User satisfaction with the Family Health Program in Vespasiano, Minas Gerais, Brazil. Rev Panam Salud Pública 2012; 31(6):454-460.

12. Pinto HÁ, Souza ANA, Ferla AA. O Programa Nacional de Melhoria do Acesso e da Qualidade da Atenção Básica: várias faces de uma política inovadora. Saúde Debate 2014; 38:358-372.

13. Fausto MCR, Giovanella L, Mendonça MHM, Seidl H, Gagno J. The position of the Family Health Strategy in the health care system under the perspective of the PMAQ-AB participating teams and users. Saúde Debate 2014; 38(esp.):13-33.

14. Santos SMS, Oliveira VAC, Oliveira RAC, Guimarães EAA. Estratégia saúde da família: qualidade da assistência sob a perspectiva da satisfação do usuário. Rev Min Enferm 2010; 14(4):499-508.

15. Perez LG, Sheridan JD, Nicholls AY, Mues KE, Saleme PS, Resende JC, Ferreira JA, Leon JS. Professional and community satisfaction with the Brazilian family health strategy. Rev Saude Publica 2013; 47(2):403413.
16. Al-Windi A. Determinants of medicine use in a Swedish primary health care practice population. Pharmacoepidemiol Drug Saf 2005; 14(1):47-51.

17. Ali FM, Nikoloski Z, Reka H. Satisfaction and responsiveness with health-care services in Qatar - evidence from a survey. Health Policy 2015; 119(11):1499-505.

18. Singh H, Haqq ED, Mustapha N. Patients' perception and satisfaction with health care professionals at primary care facilities in Trinidad and Tobago. Bull WHO 1999; 77(4):356-360.

19. Poot AJ, den Elzen WP, Blom JW, Gussekloo J. Level of Satisfaction of Older Persons with Their General Practitioner and Practice: Role of Complexity of Health Problems. Plos One 2014; 9(4):e94326.

20. Yousif N, Hussain HY, Mhakluf MMED. Health care services utilization and satisfaction among elderly in Dubai, UAE and some associated determinants. Models Systems Elderly Care 2014; 11(3):25-33.

21. Albuquerque MSV, Farias SF, Mendes MFM, Martelli PJL, Lyra TM. Accessibility to health services: an analysis of the Primary Care in the state of Pernambuco. Saúde Debate 2014; 38(esp.):182-194.

22. Amaral FLJS, Motta MHA, Alves SB. Fatores associados com a dificuldade no acesso de idosos com deficiência aos serviços de saúde. Cien Saúde Coletiva 2012; 17(11):2991-3001.

23. Alves MGM, Casotti E, Oliveira LGD, Machado MTC, Almeida PF, Corvino MPF, Marin J, Flauzino RF, Montenegro LAA. Factors affecting access to the Family Health Strategy teams in Brazil. Saúde Debate 2014; 38(esp.):34-51.

24. Paim J, Travassos C, Almeida, C, Bahia L, Macinko J. O sistema de saúde brasileiro: história, avanços e desafios. Lancet 2011; 11-31.

25. Santa Helena ET, Andersen SE, Menoncin SM. Percepção dos usuários sobre acesso aos medicamentos na atenção primária. Cad Saúde Colet 2015; 23(3):280-288.

26. Alvares J, Almeida AM, Araújo VE, Ascef BO, Costa KS, Costa EA, Soeiro OM, Leite SN, Karnikownski MG, Guibu IA, Acurcio FD, Guerra-Júnior AA. Pesquisa Nacional de Acesso a Medicamentos no Brasil - Percepção dos Usuários da Atenção Básica do Sistema Único de Saúde. Value Health 2015; 18(7):A849.

27. Caprara A, Rodrigues J. Asymmetric doctor-patient relationship: rethinking the therapeutic bond. Cien Saude Colet 2004; 9(1):139-146.

28. Coutinho AT, Popim RC, Carregã K, Spiri WC. Integralidade do cuidado com o idoso na estratégia de saúde da família: visão da equipe. Esc Anna Nery 2013; 17(4):628-637.

29. Albuquerque ABB, Bosi MLM. Home visits as a component of the Family Health Program: user's perceptions in Fortaleza, Ceará State, Brazil. Cad Saude Publica 2009; 25(5):1103-1112.

Artigo apresentado em 06/08/2018

Aprovado em 10/12/2018

Versão final apresentada em 12/12/2018 